\title{
High Speed Measurement with EtherCAT and PC based Condition Monitoring
}

\author{
Dresselhaus, Pascal \\ Beckhoff Automation $\mathrm{GmbH}$ \\ Eiserstraße 5, 33415 Verl, Germany
}

The recording of the current machine status is increasingly important in regard to reduction of unplanned standstill times, the associated costs of failure and the optimisation of maintenance intervals. A Condition Monitoring System (CMS) allows condition-orientated maintenance by means of precise measurement technology and mathematical algorithms. The probability of an unexpected machine failure can be drastically reduced with an intelligent early warning system. This not only drastically cuts the costs, but also allows better planning and preparation of maintenance work on a machine.

Classic Condition Monitoring Systems are often very expensive systems with their own CPU, software and bus systems as well as specially adapted I/Os. Powerful industry PCs, intelligent control software and the fast and deterministic EtherCAT fieldbus system allows the integration of a Condition Monitoring System into the PC-based machine controller. Alongside cost savings it enables the recording and analysis of vibration data taking full account of the operating state of the corresponding machine.

\section{EtherCAT as a measurement bus}

EtherCAT has established itself as a measurement technology standard and has been used all over the world by many end users, including some in test bench technology. The excellent performance of EtherCAT is often named as the main reason for this. In the end EtherCAT is not only several orders of magnitude faster than traditional measurement buses, but also the fastest among the industrial Ethernet solutions. The reason is the good utilisation of bandwidth, which is guaranteed by the EtherCAT functional principle, processing in passing. So utilisation data rates of well over $90 \%$ with full duplex Fast Ethernet and bus cycle times of $100 \mu$ s are possible. Together with oversampling the sampling rates can be raised well above the actual bus speed. For example digital input terminals can sample signals of up to $1 \mathrm{MHz}$ by oversampling. By the same method analog signals of up to $100 \mathrm{kHz}$ can be sampled.

A further argument for EtherCAT as measurement bus is its very high precision. This is achieved by clocks distributed in the EtherCAT slaves, which record the time-synchronised measured values across the network. The jitter which occurs is significantly smaller than a microsecond, in fact, mostly under 100 nanoseconds. The time stamp for input and output data possess the same high accuracy, which the digital time stamp of the EtherCAT bus terminals also makes use of. Some input and output terminals can exactly provide with a rising or falling edge or specify switching times by means of network-wide synchronisation and the precise time stamp.

EtherCAT systems also score points for their low costs: For one, by a software-implemented master on standard Ethernet ports and for the other by cost-effective slave controllers. Additionally, no switches are required. Time-consuming network tuning is not required in the area of engineering. The diagnostic features and the cable redundancy capability of EtherCAT allow exact error localisation, which in turn saves time and costs. EtherCAT simplifies applications by a large variety of fieldbus gateways. Existing devices can be connected into the EtherCAT network and interfaces can be implemented with neighbouring or higher-level systems. The complex variety of interfaces in central controllers with a connection over $\mathrm{PCl}, \mathrm{cPCl}, \mathrm{PCle}$ and so on, is no longer required. The support of many different fieldbuses is an important factor for the acceptance of EtherCAT in measurement technology, as well as in all other areas. The highly flexible topologies, which can be implemented with EtherCAT, are practically not subject to any limits: Line, star, tree, redundant ring or combinations of the topologies described are possible. 65,535 nodes can be used per segment. For a device spacing of more than $100 \mathrm{~m}$ the user can make use of optical fibres. Hot Connect represents an important criterion for flexible test benches. Hot Connect allows the coupling and uncoupling of build groups to the fieldbus during operation. New on the market are EtherCAT Bus Terminals for the direct connection of accelerometers (piezo vibration sensors) with IEPE interfaces (Integrated Electronics Piezo Electric). Thus, expensive analog converters are not needed. For example, Beckhoff Automation offer a product with oversampling function and a frequency range of $0.05 \mathrm{~Hz}$ to $50 \mathrm{kHz}$. 
With bus terminals like this, very modular and flexible applications can be created. Special I/Os for a Condition Monitoring system can be placed right next to I/Os for the standard machine controller. You can use a common fieldbus infrastructure.

\section{Modular analysis software in the controller}

The modular approach of the hardware is also carried on in the software. So there is a Condition Monitoring software library from Beckhoff which makes various mathematical algorithms available in the form of individual modules. These modules can be combined in almost any fashion and thus allow chains of measurement and analysis which are individually adapted to the application. These analysis chains can be directly programmed in IEC 611313 on the PLC, which in most cases more or less takes over the control of the machine. As it is possible to use a common software platform for the control and monitoring of the application, this significantly reduces the engineering effort. Also the cross-communication of the CMS required for the control in parallel systems is not needed. The programming effort which is required there is very high, above all for manufacturer-specific interfaces.

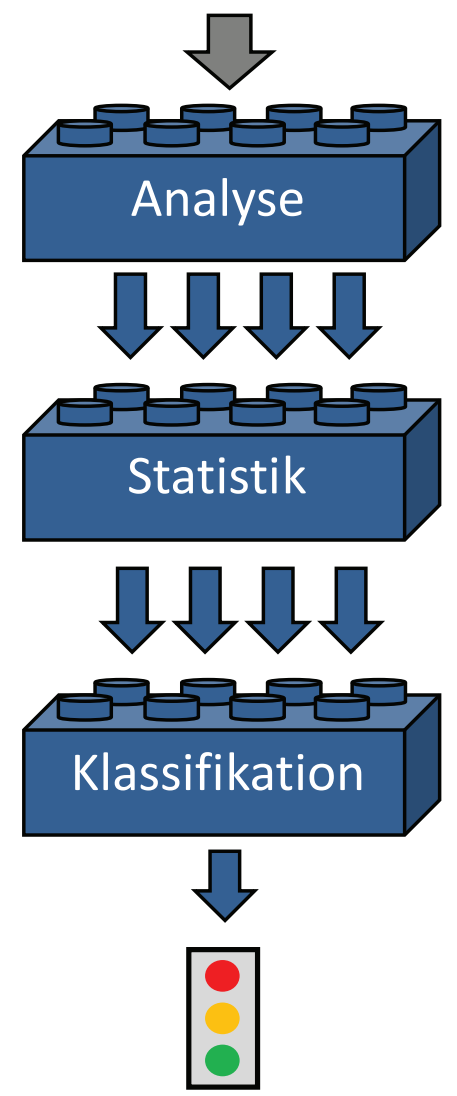

Figure 1: Modular Condition Monitoring software architecture

A further important aspect for integration of Condition Monitoring in the machine controller is the data management. While parallel systems, which often behave like a black box, only have limited access to the actual raw data, with an integrated system full data control is available. Especially for damage source analysis often the raw data helps to reconstruct the behaviour patterns observed. Data can be stored in binary data format, classic SQL databases or in open scientific data formats like netCDF or HDF5. By full data control along with the actual raw data it is also possible to save the pre-processed data, that is the results of one or more algorithms. 


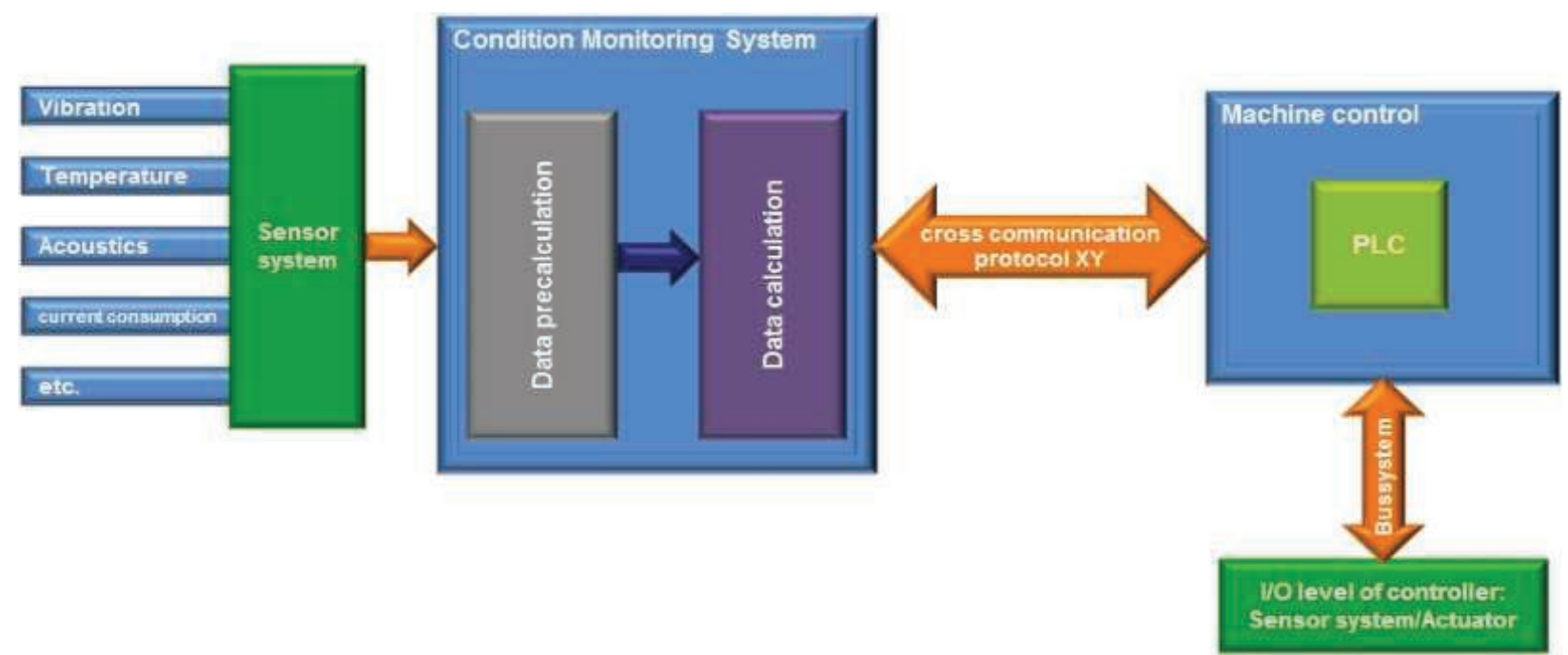

Figure 2: Measuring and analysis chain for parallel systems with special hardware and cross communication

The library algorithms are programmed in their modules, so that the user only has to parameterise them. The functionality is sub-divided into the main fields of analysis, statistics and classification. From the analysis area the user can add Fast Fourier Transformations, various window functions, digital filters, the Hilbert Envelope or the Cepstrum into their application. The result can be further statistically evaluated by considering the Standard Deviation, the Kurtosis, the Percentile or for example, by the Crest Factor. In the classification area algorithms for adaptation and monitoring of limit values and practical procedures for automatic pattern recognition are implemented.

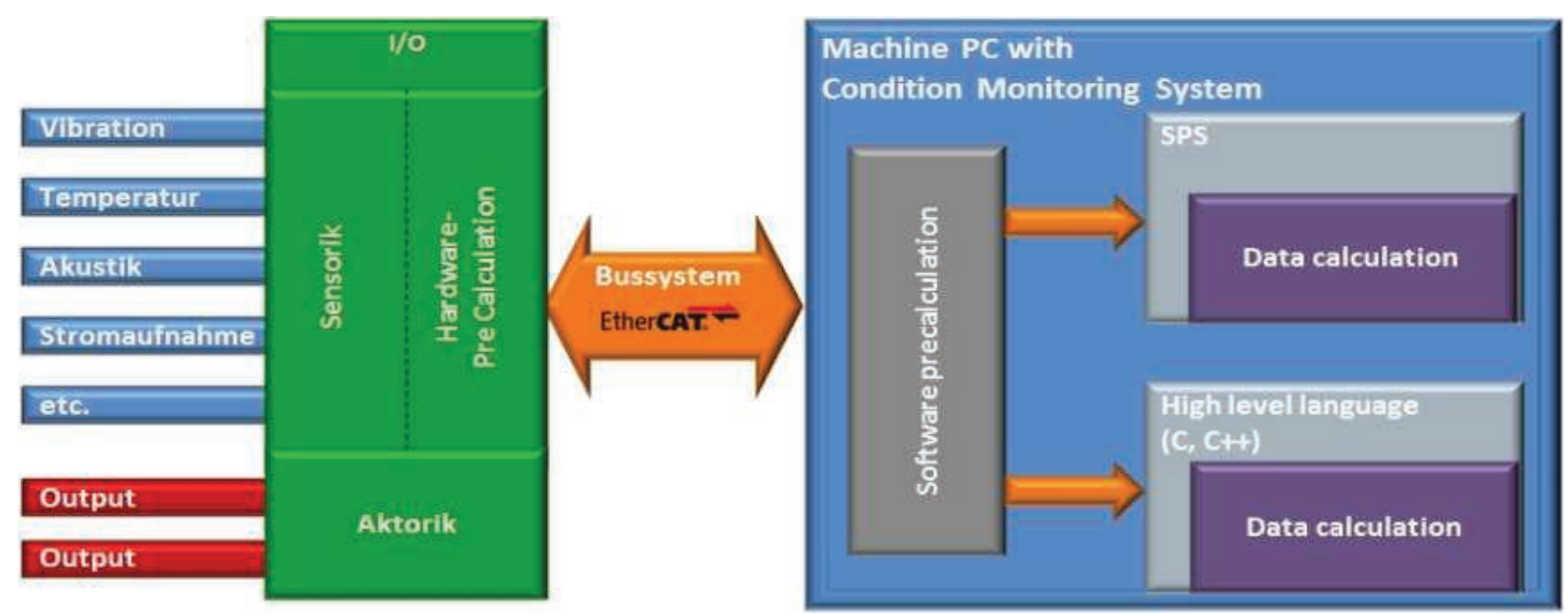

Figure 3: Integration of Condition Monitoring into the machine IPC with standard I/Os

\section{Presentation of a roller bearings monitoring system concept}

Decisive for the signal analysis as well as the tools, the software algorithms and the modular hardware for connecting the sensors, is an integrated analysis concept, which is adapted to the application instance. A common scenario is the monitoring of roller bearings, that belong to the machine elements which are under the greatest strain. In many cases they have a critical significance for the operation of a machine. The most common damage patterns are heavy wear of the inside and outside ring surfaces as well as damage to the rolling elements. The causes are numerous: As well as material fatigue, incorrect lubrication, corrosion, contamination, incorrect alignment and overloading are often causes of damage. In order to meet the requirements of a high machine availability and an efficient machine maintenance, Beckhoff offers an integrated solution with the EtherCAT vibration measurement terminal and the TwinCAT Condition Monitoring library which is scalable on different PC platforms. Three software library algorithms are particularly suited to monitoring of roller bearings: the Crest Factor, the Kurtosis and the Envelope Spectrum. 


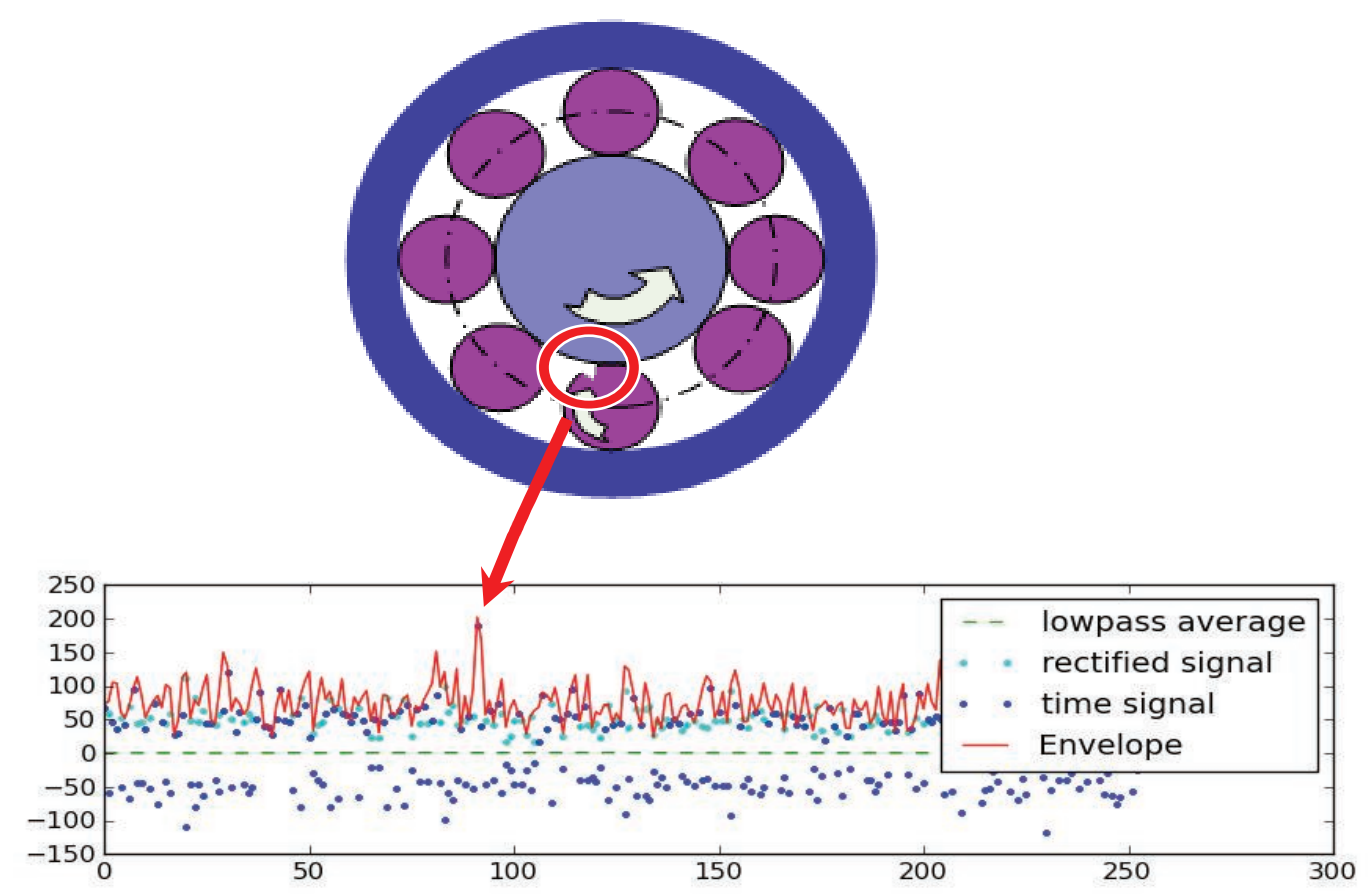

Figure 4: Damage bearing with Envelope Spectrum analysis

In the statistics area, the Crest Factor and the Kurtosis are important. The former gives the so-called Crest Factor in the vibration signal, this means that the relationship between maximum amplitude and the effective measured, mean vibration amplitude is calculated. An increase in this factor points to a high probability of damage. The Crest Factor is a simple-to-interpret parameter and is well suited to trend analysis. A disadvantage is that the Crest Factor does not represent a robust parameter in a statistical sense. In comparison to this, the Kurtosis is much more robust, as it gives a weighted relationship between the extreme deviations, from the mean value of a distribution and the mean fluctuation range of the vibration amplitude and thus is not dependent on individual maximum values. The greater the value of the Kurtosis, the steeper the distribution, which points to a damage. In contrast to both parameters already explained, the Envelope Spectrum comes from the area of vibration analysis. The calculation is more complex, in fact due to the spectrum, even individual defective roller bearing elements can be identified. This knowledge is secondary in many applications and requires a lot of know-how in relation to the bearing geometry. In the foreground are the general good damage detection options offered by the Envelope Spectrum. The defect recognition is due to the evaluation of pulsed shocks, which are caused by damage on the contact surfaces. Damage to a rotating part results in the periodic shock pulse. The rotational speed and the geometry of the roller bearing elements decide the period of the shock pulse, which allows identification of the defective component. The Envelope Spectrum is then searched for certain periodically recurring spectral lines, whereby the period itself identifies the defective part.

To further evaluate the crest factor and Kurtosis statistical parameters presented here, the software library allows the definition of limit values and offers further classification algorithms. Various warning and alarm levels can be set and monitored for differing machine elements. This allows the user the best match of machine maintenance intervals to the operational production plan. In general, a good monitoring concept offers the possibility of early damage recognition, as well as an instrument for the identification of individual elements involved, by the analysis of vibration signals. The earlier damage can be recognised and limited to individual elements, the greater the probability not just of avoiding an unexpected machine failure, but also of drawing conclusions regarding the initial damage. The experience which has been gained can be channelled straight back into the construction of new machines.

\section{Conclusions}

The performance of the current generation of PCs allows the integration of Condition Monitoring in the PC-based controller. Future multi-core processors will extend this approach. EtherCAT provides a field bus which is not just fast and deterministic, but is also a globally-accepted standard. The EtherCAT Technology Group (ETG) represents the largest industrial Ethernet organisation in the world with over 
1300 members in 45 countries. With the analysis functions implemented in the software libraries various applications, from a so-called traffic light solution, in which the user can easily interpret the condition of the machine by green, yellow and red signal colours, to a complex analysis platform with damage source detection, can be set up. By modular integration in the hardware and software the possible applications are extremely scalable and flexible, whereby Condition Monitoring is available even for the smallest machines for a reasonable price. 